\title{
Effective Noise Cancellation Using Single-Carrier FDMA Transmission in Power-Line Channels
}

\author{
Khaled M. Rabie, Student Member, IEEE, Emad Alsusa, Senior Member, IEEE
}

\begin{abstract}
In power-line channels impulsive noise (IN) is commonly reduced using a blanking technique in which the received signal is zeroed whenever it exceeds a certain threshold value. Determining an appropriate threshold however is critical to the performance of this technique as erroneously blanking unaffected parts of the signal will significantly degrade the system performance. In this paper, we show that the performance of this technique is sensitive not only to the blanking threshold but also to the transmitted signal's peak-to-average power ratio (PAPR). With this in mind we propose to implement single-carrier FDMA (SC-FDMA), which inherently has low PAPR, and address the issue of optimizing the blanking threshold for such technique. The results reveal that significant improvements can be achieved with respect to the probability of blanking error, as well as achieving up to $4 \mathrm{~dB}$ SNR improvement relative to the conventional OFDM case. Furthermore, it will be shown that in some cases of SCFDMA, not only a significant SNR enhancement can be achieved but also it is possible to optimally blank IN irrespective of the noise characteristics.
\end{abstract}

Index Terms-Blanking, impulsive noise, interleaved FDMA (IFDMA), localized FDMA (LFDMA), power-line communications (PLC), signal-to-noise ratio (SNR), single-carrier frequency division multiple access (SC-FDMA).

\section{INTRODUCTION}

$\mathbf{P}$ OWER-LINE communication (PLC) is continuing to be a more attractive alternative for inhome networking applications providing communications at $200 \mathrm{Mb} / \mathrm{s}$ [1]. The prominent advantage of PLC networks is the fact that they utilize an existing infrastructure of wiring networks which can be easily accessed through electricity outlets in the home. This technology becomes even more appealing in harsh wireless environments where propagation loss is high such as in underground structures and buildings with metal walls [2]. However, such medium does not represent a favorable channel for communication signals since power lines have never been designed for signal transmission at high frequency. For reliable communications over power lines, it is of utmost importance to overcome few obstacles including the strong branching problem which considerably affects the signal quality with a large number of reflection points [3], crosstalk between the wires, noise [4]-[6] and high levels of frequency-dependent attenuation [7]. Unlike conventional communication channels, noise over power lines can not be represented as additive white Gaussian noise (AWGN). It is rather categorized into background noise (BN) and impulsive noise (IN) [4], [8]. The latter is, however, the most dominant factor responsible for degrading communication signals [9]. IN has a short duration with random occurrence

The authors are with the School of Electrical \& Electronic Engineering, The University of Manchester, Manchester, M13 9PL, UK. (e-mails: \{khaled.rabie, e.alsusa\}@manchester.ac.uk). rate and high power spectral density (PSD) [4], [10]. IN can have durations frequently exceeding the signal symbol length which can seriously affect high speed communications [11]. To analyze and evaluate the system performance in the presence of IN, Middleton class-A noise model, [12], [13], has been widely accepted and therefore it will be adopted in this paper.

Multicarrier modulation such as orthogonal frequency division multiplexing (OFDM) systems [14] have been proposed for PLC in [15] and adopted by many PLC standards [1]. Several methods have been reported in the literature on the topic of mitigating IN in power-line OFDM based systems. The simplest of which is to precede the OFDM demodulator with a blanker to zero the incoming signal when it exceeds a certain threshold [16]-[20]. In these methods, the selection of blanking threshold is the key for achieving best performance. Theoretical performance analysis and blanking threshold optimization is considered in [18], [20] where closed-form expressions for the signal-to-noise ratio (SNR) at the output of the blanker and the optimal blanking threshold (OBT) were derived. These studies rely on the assumption that the IN characteristics, in the form of signal-to-impulsive noise ratio (SINR) and the IN probability of occurrence, can be made available at the receiver in order to optimally blank IN. Such assumptions, however, may be difficult to fulfill in practice because of the short-term variations of IN. In such scenario, estimation errors of noise parameters could lead to imperfect recognition of the IN signal. As a result, this will cause blanking uncorrupted signal samples causing blanking errors and hence significant performance deterioration [21]. Not only that, but also uncorrupted signal samples with high amplitude may wrongly trigger the blanker causing errors [22]. This can be the case in OFDM systems as they suffer from high peak-to-average power ratio (PAPR). Therefore, in this study we show that the performance of blanking-based IN mitigation technique is sensitive not only to the blanking threshold but also to the signal's PAPR. In contrast to other studies, in this paper we propose to implement single-carrier frequency-division multiple access (SC-FDMA), which inherently has low PAPR [23], [24], and address the issue of optimizing the blanking threshold to enhance the overall performance of the system. Two SC-FDMA schemes are considered in this work namely, Localized FDMA (LFDMA) and Interleaved FDMA (IFDMA).

The contribution of this paper is threefold. First we begin with investigating the PAPR of the OFDMA, LFDMA and IFDMA systems and then demonstrate how such signals' peaks can affect the blanking threshold. Then, two different quantitative characterization aspects of the achievable performance are considered. These aspects are the probability of blanking error and the SNR at the output of the blanking device. The third con- 


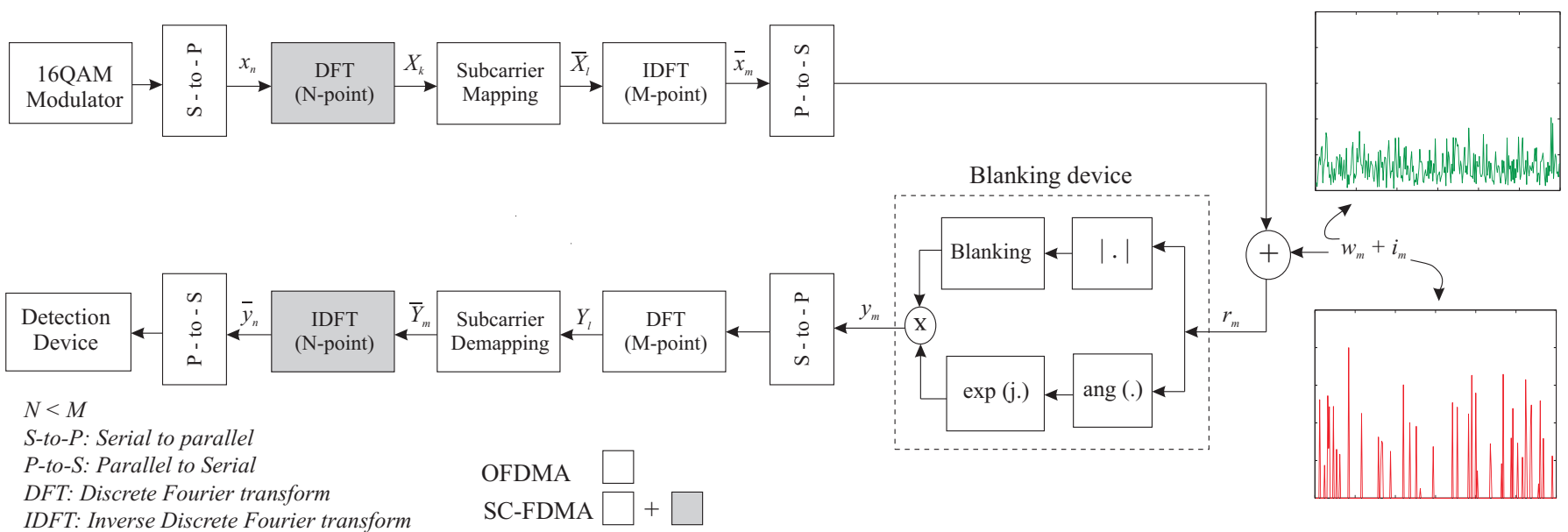

Fig. 1: SC-FDMA system with a blanking nonlinear preprocessor at the receiver.

tribution resides in addressing the problem of blanking threshold optimization and the corresponding maximum achievable output SNR of the aforementioned systems. The results reveal that the SC-FDMA with blanking can significantly minimize the probability of blanking error and is able to provide about $4 \mathrm{~dB}$ SNR enhancement relative to the conventional OFDMA systems. Furthermore and most importantly, it will be shown that when IFDMA is utilized, the OBT becomes independent of the IN characteristics. This implies that the proposed not only outperforms the conventional OFDMA systems in terms of output SNR but also can completely alleviate the need for any prior knowledge about the IN characteristics in order to optimally blank the noise.

The rest of this paper is organized as follows. In Section II the system model is described. Section III presents a comparative analysis of the PAPR for OFDMA, LFDMA and IFDMA systems and their relationship with the blanking threshold. In Section IV, the probability of blanking error for the OFDMA, LFDMA and IFDMA systems is investigated and the corresponding output SNR performance is presented in Section $\mathrm{V}$. The problem of blanking threshold optimization of the three FDMA systems and the corresponding maximum achievable output SNR are addressed in Section VI. Finally, conclusions are drawn in Section VII.

\section{System Model OVERVIEW}

The basic system model used in this study is shown in Fig. 1. First the information bits are mapped into baseband 16QAM symbols which are then grouped into blocks each of length $N$ symbols by the serial to parallel (S-to-P) converter, $\left\{x_{n}: n=0,1, \ldots, N-1\right\}$. After that these blocks are passed through an $N$-point discrete Fourier transform (DFT) modulator to produce the frequency domain representation, $\left\{X_{k}: k=0,1, \ldots N-1\right\}$ which is defined as

$$
X_{k}=\sum_{n=0}^{N-1} x_{n} e^{\frac{-j 2 \pi n k}{N}}
$$

$X_{k}$ is then fed to the subcarrier mapping device to produce $\left\{\bar{X}_{l}: l=0,1, \ldots, M-1\right\}$ where $M>N$. Several mapping patterns have been introduced in the literature such as LFDMA and the IFDMA [23], [24]. The principle of these mapping techniques are presented in Fig. 2 for $M=16$ and $N=4$; the ratio $Q=N / M$ denotes the band spreading factor. In the first scheme, consecutive subcarriers are occupied by the DFT outputs with zeros occupying the remaining subcarriers such that

$$
\bar{X}_{l}= \begin{cases}X_{l}, & 0 \leq l \leq N-1 \\ 0, & N \leq l \leq M-1\end{cases}
$$

Whereas in the IFDMA scheme, the DFT outputs are allocated over the entire bandwidth with equal distance while zero padding the unused subcarriers

$$
\bar{X}_{l}= \begin{cases}X_{l / Q}, & l=Q \cdot k \quad(0 \leq k \leq N-1) \\ 0, & \text { Otherwise }\end{cases}
$$

The frequency domain samples $\bar{X}_{l}$ are then passed through an $M$-point inverse discrete Fourier transform (IDFT) modulator to produce $\bar{x}_{m}$, given by (4), before going into the parallel to serial (P-to-S) convertor and then transmission.

$$
\bar{x}_{m}=\frac{1}{M} \sum_{l=0}^{M-1} \bar{X}_{l} e^{\frac{j 2 \pi m l}{M}}
$$

Using the notation in (4), the PAPR of the transmitted signal can be expressed as

$$
\text { PAPR }=10 \log _{10}\left(\frac{\max _{m=0,1, \ldots, M-1}\left|\bar{x}_{m}\right|^{2}}{\frac{1}{M} \sum_{m=0}^{M-1}\left|\bar{x}_{m}\right|^{2}}\right)
$$

This paper adopts the Middleton class-A model with probability density function (PDF)

$$
p(z)=\sum_{i=0}^{\infty} \frac{e^{-A} A^{i}}{i !} \cdot \frac{1}{\sqrt{2 \pi \sigma_{i}^{2}}} e^{\left(-\frac{z^{2}}{2 \sigma_{i}^{2}}\right)}
$$




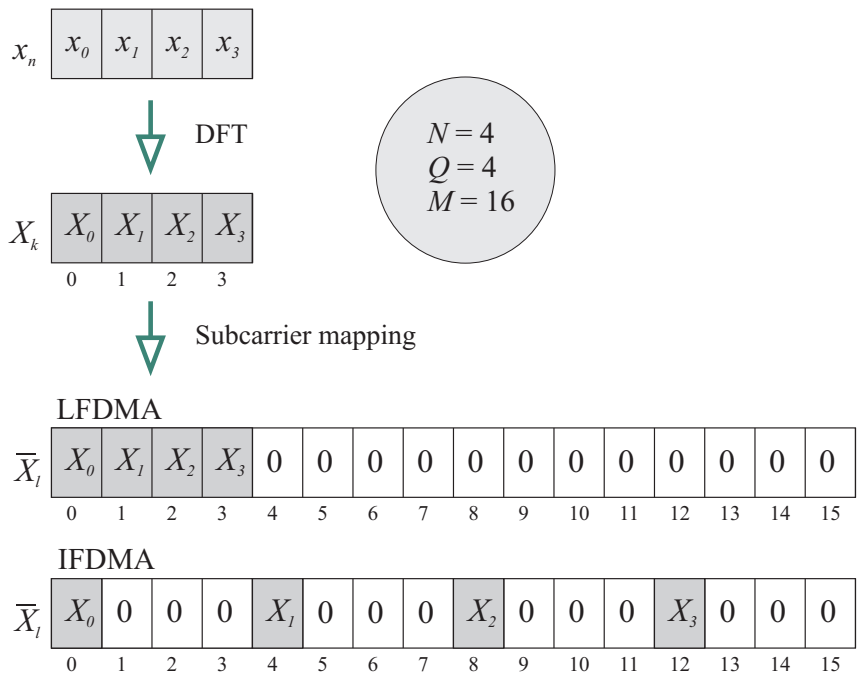

Fig. 2: The frequency domain symbols for LDFMA and IFDMA subcarrier mapping schemes when $N=4, Q=4$ and $M=16$.

where

$$
\begin{gathered}
\sigma_{i}^{2}=\sigma^{2}\left(\frac{\frac{i}{A}+\Gamma}{1+\Gamma}\right) \\
\sigma^{2}=\sigma_{G}^{2}+\sigma_{I}^{2} \\
\Gamma=\frac{\sigma_{G}^{2}}{\sigma_{I}^{2}}
\end{gathered}
$$

While $A$ measures the average number of impulses over the signal period and is referred to as impulsive index, $\sigma^{2}$ is the total noise power, $\sigma_{G}^{2}$ is the Gaussian noise power and $\sigma_{I}^{2}$ is the impulsive (non-Gaussian) noise power. For the sake of simplicity, in this work a special case of Middleton class-A noise model is deployed in which IN is modeled as a BernoulliGaussian random process [25] and is given as

$$
n_{m}=w_{m}+i_{m}, \quad m=0,1,2, \ldots, M-1
$$

where

$$
i_{m}=b_{m} g_{m}, \quad m=0,1,2, \ldots, M-1
$$

$n_{m}$ is the total noise component, $w_{m}$ is the AWGN, $i_{m}$ is the IN, $g_{m}$ is complex white Gaussian noise with mean zero and $b_{m}$ is the Bernoulli process with probability mass function

$$
\operatorname{Pr}\left(b_{m}\right)=\left\{\begin{array}{ll}
p, & b_{m}=1 \\
0, & b_{m}=0
\end{array} \quad m=0,1, \ldots, M-1\right.
$$

where $p$ is referred to as the IN probability of occurrence. The PDF of the total noise can be expressed as

$$
P_{n_{m}}\left(n_{m}\right)=(1-p) \mathcal{G}\left(n_{m}, 0, \sigma_{w}^{2}\right)+p \mathcal{G}\left(n_{m}, 0, \sigma_{w}^{2}+\sigma_{i}^{2}\right)
$$

$\mathcal{G}($.$) is the Gaussian PDF and is given by (14). \sigma_{w}^{2}$ and $\sigma_{i}^{2}$ are the AWGN and IN variances which define the input SNR and SINR as in (15) and (16), respectively, where $\sigma_{\bar{x}}^{2}$ is the variance of the transmitted signal.

$$
\begin{gathered}
\mathcal{G}\left(s, \mu, \sigma_{s}^{2}\right)=\frac{1}{\sqrt{2 \pi \sigma_{s}^{2}}} e^{-\frac{(s-\mu)^{2}}{2 \sigma_{s}^{2}}} \\
\mathrm{SNR}=10 \log _{10}\left(\frac{\sigma_{\bar{x}}^{2}}{\sigma_{w}^{2}}\right) \\
\mathrm{SINR}=10 \log _{10}\left(\frac{\sigma_{\bar{x}}^{2}}{\sigma_{i}^{2}}\right)
\end{gathered}
$$

Assuming perfect synchronization, the received signal is expressed as

$$
r_{m}=\left\{\begin{array}{ll}
\bar{x}_{m}+w_{m}, & \mathcal{H}_{0} \\
\bar{x}_{m}+w_{m}+i_{m}, & \mathcal{H}_{1}
\end{array} \quad m=0,1, \ldots, M-1\right.
$$

$\bar{x}_{m}, w_{m}$ and $i_{m}$ are assumed to be mutually independent. The null hypothesis $\mathcal{H}_{0}$ implies the absence of IN, $P\left(\mathcal{H}_{0}\right)=$ $(1-p)$, whereas the alternative hypothesis $\mathcal{H}_{1}$ implies the presence of IN, $P\left(\mathcal{H}_{1}\right)=p$. In order to reduce the energy of IN, a blanking nonlinear preprocessor is applied at the frontend of the receiver (before the SC-FDMA demodulator) as illustrated in Fig. 1. The output of this device is given as

$$
y_{m}=\left\{\begin{array}{ll}
r_{m}, & \left|r_{m}\right| \leq T \\
0, & \left|r_{m}\right|>T
\end{array} \quad m=0,1, \ldots, M-1\right.
$$

where $r_{k}$ and $y_{k}$ are the input and output of the blanker, respectively, and $T$ is the blanking threshold. It is noted that the device only processes the amplitude of the received signal without modifying its phase. The selection of the threshold value is the key for obtaining best performance. For instance, if the threshold is too small, many unaffected samples of the useful transmitted signal will be blanked resulting in poor bit error rate performance. On the other hand, for very large threshold, IN will be overlooked and will become part of the detected signal hence will degrade performance. In [18], a theoretical expression for the OBT $\left(T_{o p t}\right)$ of the OFDM system was derived as a function of IN parameters (19) as well as the output SNR given as

$$
\operatorname{SNR}_{O F D M A}=\frac{2}{\mathbb{E}\left[A_{n}^{2}\right]}
$$

$\mathbb{E}\left[A_{n}^{2}\right]$ is defined as in (21). These expressions will be used to provide a comparative analysis to show the superiority of the proposed system over the conventional OFDM blanking-based system and also to verify the accuracy of our simulation model.

After the blanking device, the received signal $y_{m}$ is passed through the $M$-point DFT to produce $\left\{Y_{l}: l=0,1, \ldots M-1\right\}$ and the corresponding signal after the subcarrier demapping device is $\left\{\bar{Y}_{m}: m=0,1, \ldots N-1\right\}$. Finally, the signal after the $N$-point IDFT is given as $\left\{\bar{y}_{n}: n=0,1, \ldots, N-1\right\}$. 


\begin{tabular}{|l|c|c|c|c|c|c|}
\hline & \multicolumn{2}{|c|}{ OFDMA } & \multicolumn{2}{c|}{ LFDMA } & \multicolumn{2}{c|}{ IFDMA } \\
\hline \hline & $\begin{array}{c}\text { Recognized } \\
\text { IN pulses }\end{array}$ & $\begin{array}{c}\text { Signal } \\
\text { blanking errors }\end{array}$ & $\begin{array}{c}\text { Recognized } \\
\text { IN pulses }\end{array}$ & $\begin{array}{c}\text { Signal } \\
\text { blanking errors }\end{array}$ & $\begin{array}{c}\text { Recognized } \\
\text { IN pulses }\end{array}$ & $\begin{array}{c}\text { Signal } \\
\text { blanking errors }\end{array}$ \\
\hline$T_{O F D M A}$ & 2 & 0 & 2 & 0 & 2 & 0 \\
\hline$T_{L F D M A}$ & 4 & 4 & 4 & 0 & 4 & 0 \\
\hline$T_{I F D M A}$ & 6 & 9 & 6 & 3 & $\mathbf{6}$ & 0 \\
\hline
\end{tabular}

Table I: Blanking errors of OFDMA, LFDMA and IFDMA systems

$$
T_{o p t}=\sqrt{\frac{2\left(1+\sigma_{w}^{2}\right)\left(1+\sigma_{w}^{2}+\sigma_{i}^{2}\right)}{\sigma_{i}^{2}} \ln \left(\left[\frac{1+\sigma_{w}^{2}+\sigma_{i}^{2}}{1+\sigma_{w}^{2}}\right]^{2} \frac{\left(1-\sigma_{w}^{2}\right)}{\left(1-\sigma_{w}^{2}-\sigma_{i}^{2}\right)} \frac{(p-1)}{p}\right)}
$$

$$
\begin{aligned}
\mathbb{E}\left[A_{n}^{2}\right]=2(1-p)\left[\sigma_{w}^{2}\left(1-\sigma_{w}^{2}\right)\right. & \left(\frac{T^{2}}{2\left(1+\sigma_{w}^{2}\right)}+1\right) e^{\left.-\frac{T^{2}}{2\left(1+\sigma_{w}^{2}\right)}\right]} \\
+ & 2 p\left[\left(\sigma_{w}^{2}+\sigma_{i}^{2}\right)+\left(1-\sigma_{w}^{2}-\sigma_{i}^{2}\right)\left(\frac{T^{2}}{2\left(1+\sigma_{w}^{2}+\sigma_{i}^{2}\right)}+1\right) e^{\left.-\frac{T^{2}}{2\left(1+\sigma_{w}^{2}+\sigma_{i}^{2}\right)}\right]}\right.
\end{aligned}
$$

\section{The Relationship BETWEen PAPR AND BLANKING THRESHOLD}

In this section we discuss the impact of PAPR on the blanking threshold. It is intuitive to think that if the average PAPR of the transmitted signal symbols is reduced, then this will make IN more distinguishable from the useful transmitted signal and therefore can improve the blanking process at the receiver. One way of accomplishing this is by using an SCFDMA system such as LFDMA and IFDMA schemes.

For further clarity, an illustrative example is presented in Fig. 3 showing plots of OFDMA, LFDMA and IFDMA signals in addition to IN pulses. It must be mentioned that in order to provide a comparative analysis the IN is deliberately kept unchanged for the three schemes. Depending on the modulation scheme applied, this figure presents three different scenarios. First, in case of the OFDMA system it can be seen that when the blanking threshold $T_{\text {OFDMA }}$ is considered, two IN pulses will be recognized $\{$ IN5, IN6 $\}$ and IN1, IN2, IN3 and IN4 remain undetected which then become part of the signal fed to the OFDMA demodulator; whereas if $T_{\text {LFDMA }}$ or $T_{\text {IFDMA }}$ is used, this will allow detecting more IN pulses, however, unaffected samples will also trigger the blanker and consequently will be set to zero causing blanking errors. The second scenario is considering the LFDMA system with blanking threshold $T_{\text {LFDMA }}$ in which the blanker will be able to identify more IN pulses $\{$ IN1, IN4, IN5, IN6\}. The third scenario is when IFDMA is adopted which allows using $T_{\text {IFDMA }}$ with zero blanking errors in addition to eliminating all the IN pulses. Table (I) summaries these scenarios from which it is obvious that the IFDMA system provides the lowest blanking errors since it recognizes all the IN pulses (6 pulses) and leaves the unaffected signal samples untouched.

From Fig. 3, the amount of reduction in blanking threshold is referred to as blanking threshold gain $\left(\mathrm{BTG}_{\mathrm{L} / \mathrm{I}}=T_{\mathrm{LFDMA} / \mathrm{IFDMA}}-T_{\mathrm{OFDMA}}\right)$. It will be shown later that the higher the BTG, the more performance enhancement is

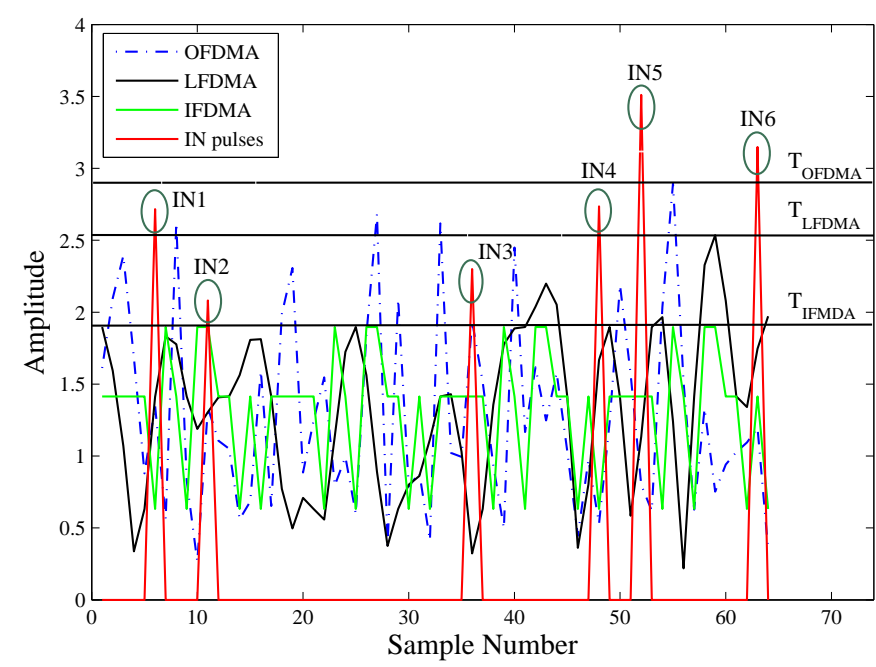

Fig. 3: An example of blanking thresholds for OFDMA, LFDMA and IFDMA when $N=16, M=64, p=0.1$ and SINR $=-6 \mathrm{~dB}$.

achieved. For better realization of the proposed system, it is important to investigate the PAPR distribution of OFDMA, LFDMA and IFDMA systems. Therefore, we introduce the complementary cumulative distribution function (CCDF) of the PAPR of these systems.

\section{A. OFDMA System}

The CCDF of PAPR is defined as the probability that the PAPR of a data block exceeds a given threshold $\left(\mathrm{PAPR}_{O}\right)$. A simple expression of the CCDF in the case of OFDMA system is derived in [26] and can be rewritten in terms of peaks $(P)$ instead of PAPR as

$$
C C D F=1-\operatorname{Pr}\left(P \leq P_{o}\right)=1-\left(1-e^{\left(-P_{o}\right)}\right)
$$




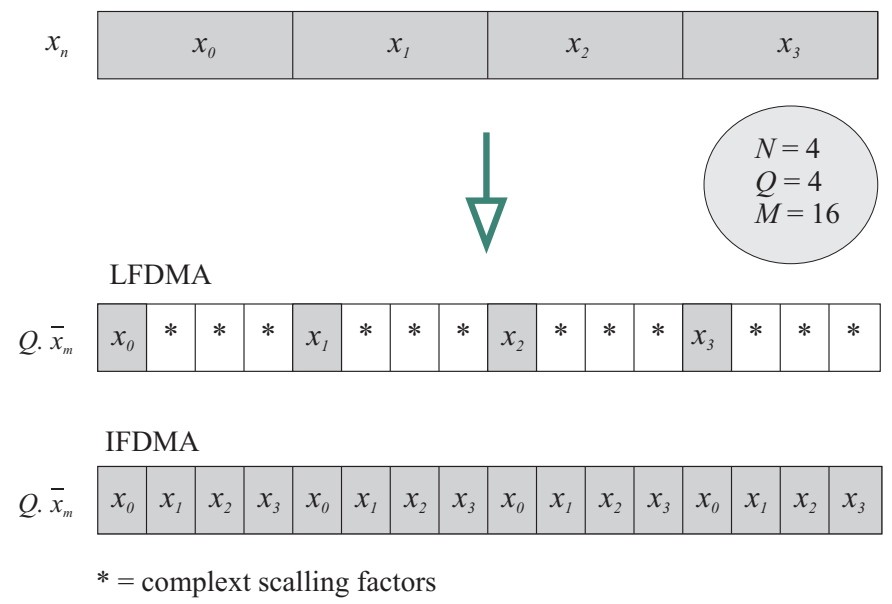

Fig. 4: Time domain symbols of LFDMA and IFDMA subcarrier mapping modes when $N=4, Q=4$ and $M=16$.

\section{B. LFDMA system}

In LFDMA, the time domain signal is obtained by substituting (2) into (4) and by letting $m=Q n+q$, where $\{n=0,1, \ldots, N-1\}$ and $\{q=0,1, \ldots, Q-1\}$, the LFDMA transmitted signal can be expressed as [27]

$$
\bar{x}_{n Q+q}= \begin{cases}\frac{1}{Q} x_{n}, & q=0 \\ \frac{\left(1-e^{j 2 \pi \frac{q}{Q}}\right)}{Q M} \sum_{p=0}^{M-1} \frac{x_{p}}{1-e^{j 2 \pi\left\{\frac{(n-p)}{M}+\frac{q}{N Q}\right\}},} & q \neq 0\end{cases}
$$

From (23), it is clear that the LFDMA transmitted signal has copies of the original sequence in $\{q=0\}$ positions with scaling factor of $1 / Q$; whereas in $q \neq 0$ positions values of the sum of all the input sequences in the input block with different complex scaling factors. This, however, increases the PAPR of the LFDMA signal.

\section{IFDMA system}

Similarly, the IFDMA transmitted signal is obtained by substituting (3) into (4). Let $m=N q+n$, where $\{n=0,1, \ldots, N-1\}$ and $\{q=0,1, \ldots, Q-1\}$, then

$$
\bar{x}_{m}\left(=\bar{x}_{N q+n}\right)=\frac{1}{Q} x_{n}
$$

It is interesting to notice from this expression that the IFDMA time domain symbols are a repetition of the input symbols $x_{n}$. This implies that the PAPR of the IFDMA signal is equal to the PAPR of the single-carrier signal. For better illustration, Fig. 4 depicts a schematic diagram of time domain signals for LFDMA and IFDMA subcarrier mapping schemes.

To compare the PAPRs of OFDMA, LFDMA and IFDMA systems, a MATLAB simulation is conducted in which 100000 random symbols are generated and the corresponding symbol peaks are calculated. Fig. 5 shows the CCDF of peaks for the three systems when 16QAM symbol constellations is used. These results are obtained for a total number of subcarriers

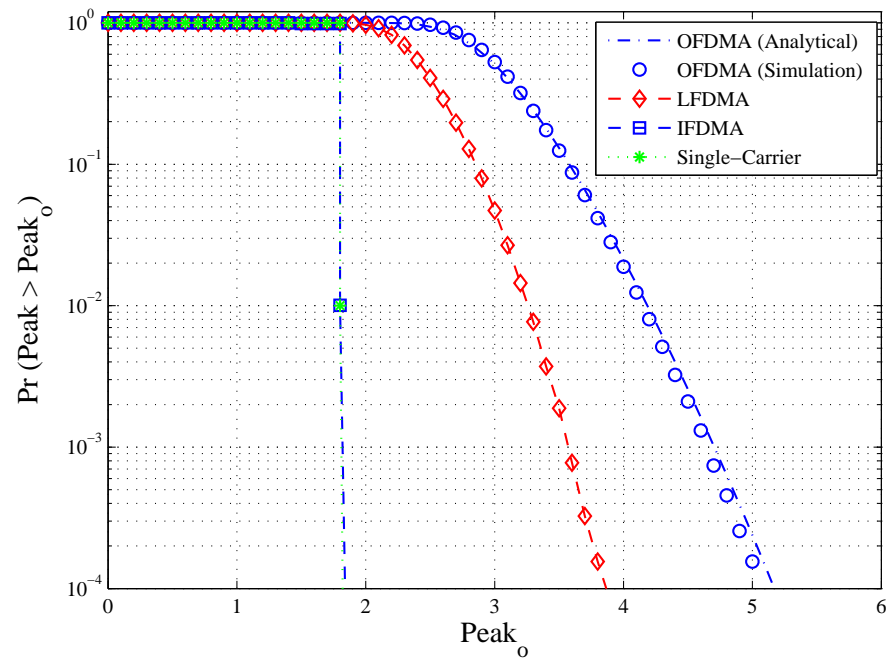

Fig. 5: CCDF for OFDMA, LFDMA and IFDMA when $M=64, N=16$ and $Q=4$.

$(M=64)$, input symbols $(N=16)$ and spreading factor $(Q=4)$. It can be seen that in the case of OFDMA the analytical results, obtained from (22), correlate well with the simulation ones. It is also observed that IFDMA consistently provides the lowest peaks with about 3.5 unites peak reduction relative to OFDMA at CCDF of $10^{-4}$ whereas about 1.5 unites of gain is obtained by LFDMA at the same CCDF value. Furthermore, it is important to point out that IFDMA has two unique properties. First and as anticipated above (24), IFDMA and single-carrier systems have exact peak distribution. The second property is the sharp drop in CCDF at around 1.9. It will be shown later that this drop is of utmost importance in determining the OBT. In general, the reduction in the peak values obtained by SC-FDMA systems implies that more of the transmitted signal energy is contained close to the average value and hence larger BTG value can be attained.

\section{The Probability of Blanking Error}

The probability of blanking error $\left(P_{b}\right)$ is defined as the probability that the amplitude of the received sample, $A_{r}=\left|r_{m}\right|$, exceeds the blanking threshold when it is unaffected by IN and it is given as $P_{b}=P\left(B, \mathcal{H}_{0}\right)$, where $B$ is the event of blanking the received signal exceeding $T$. According to Bayes' theorem, $P\left(B, \mathcal{H}_{0}\right)=P\left(B \mid \mathcal{H}_{0}\right) P\left(\mathcal{H}_{0}\right)$. Thus,

$$
P_{b}=P\left(A_{r}>T \mid \mathcal{H}_{0}\right) P\left(\mathcal{H}_{0}\right)
$$

For the OFDMA system, in the absence of IN the amplitude of the received signal has Rayleigh distribution with parameter $\sigma^{2}=\sigma_{\bar{x}}^{2}+\sigma_{w}^{2}$ and its conditional PDF can be expressed as

$$
f_{A_{r}}\left(r \mid \mathcal{H}_{0}\right)=\frac{r}{\left(\sigma_{\bar{x}}^{2}+\sigma_{w}^{2}\right)} e^{-\left(\frac{r^{2}}{2\left(\sigma_{\bar{x}}^{2}+\sigma_{w}^{2}\right)}\right)} P\left(\mathcal{H}_{0}\right)
$$

and the probability of blanking error is

$$
\begin{aligned}
P_{b} & =\int_{T}^{\infty} f_{A_{r}}\left(r \mid \mathcal{H}_{0}\right) \mathrm{dr} \\
& =e^{-\frac{T^{2}}{2\left(\sigma_{\bar{x}}^{2}+\sigma_{w}^{2}\right)}}(1-p)
\end{aligned}
$$




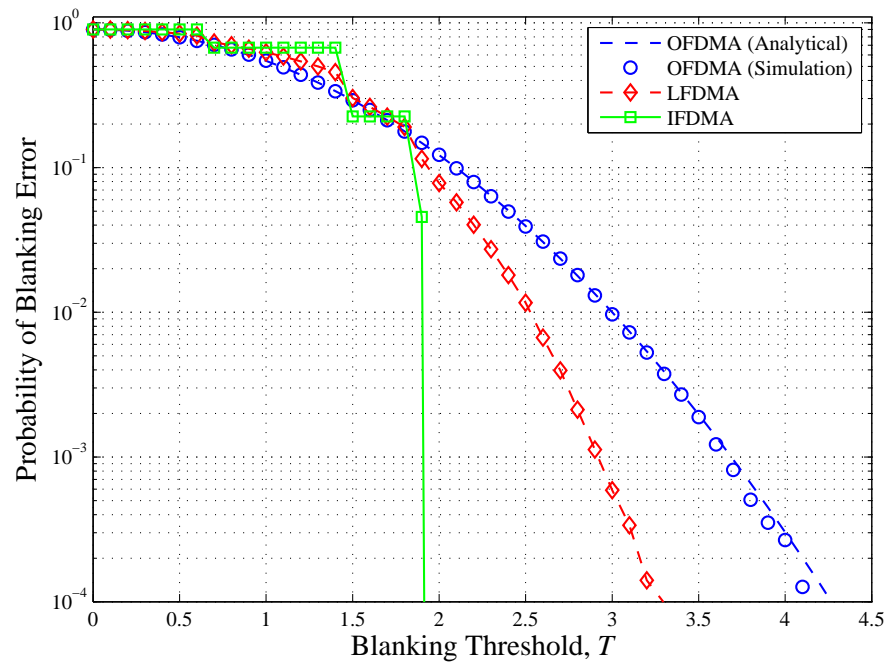

Fig. 6: Probability of blanking error versus blanking threshold for OFDMA, LFDMA and IFDMA systems, SNR $=40 \mathrm{~dB}$.

Some numerical results of (27) are shown in Fig. 6 along with simulation results for OFDMA, LFDMA and IFDMA. It is clearly observed that the analytical and simulation results of the OFDMA are matching. Our results from this point onward are based on an OFDM system consisting of $M=64$ subcarriers, $N=16, Q=4$, 16QAM modulation, $\sigma_{\bar{x}}^{2}=(1 / 2) \mathbb{E}\left[\left|\bar{x}_{m}\right|^{2}\right]=$ $1, \sigma_{w}^{2}=(1 / 2) \mathbb{E}\left[\left|w_{m}\right|^{2}\right]$ and $\sigma_{i}^{2}=(1 / 2) \mathbb{E}\left[\left|i_{m}\right|^{2}\right]$.

In general, it is evident that both LFDMA and IFDMA exhibit lower probability of blanking error in comparison with OFDMA. The behavior of the probability can be divided into two regions. The first region is $\{T \lesssim 2\}$ during which SCFDMA systems do not provide any reduction. In the second region $\{T \gtrsim 2\}$ it is noticeable that both SC-FDMA schemes have lower probability of blanking error whilst IFDMA provides the lowest probability. The general trend in this region is that as $T$ increases the probability is minimized. For instance, in the LFDMA system and at $T=2.5$, the probability is reduced by about 0.3 order of magnitude; whereas at $T=3$ the probability is minimized by about 1 order of magnitude. However, in the case of IFDMA it is interesting to note that at about $T=2$, the probability falls dramatically offering zero blanking error beyond this threshold. The reduction in the probability implies that the SNR performance will improve as will be further discussed in the next section.

\section{The OUtPut SNR Versus BLANKING THREShOLD}

In this section we analyze the performance of the proposed system, by means of simulation, in terms of SNR at the output of the blanking device which is calculated as

$$
\mathrm{SNR}_{O / L / I F D M A}=\frac{\mathbb{E}\left[\left|\bar{x}_{m}\right|^{2}\right]}{\mathbb{E}\left[\left|y_{m}-\bar{x}_{m}\right|^{2}\right]}
$$

The output SNR as a function of blanking threshold is illustrated in Fig. 7 for OFDMA, LFDMA and IFDMA when SINR $=-10 \mathrm{~dB}, p=0.01$ and SNR $=40 \mathrm{~dB}$. The reason why we chose such high SNR is to keep the focus of the results on the impact of the IN element that the proposed

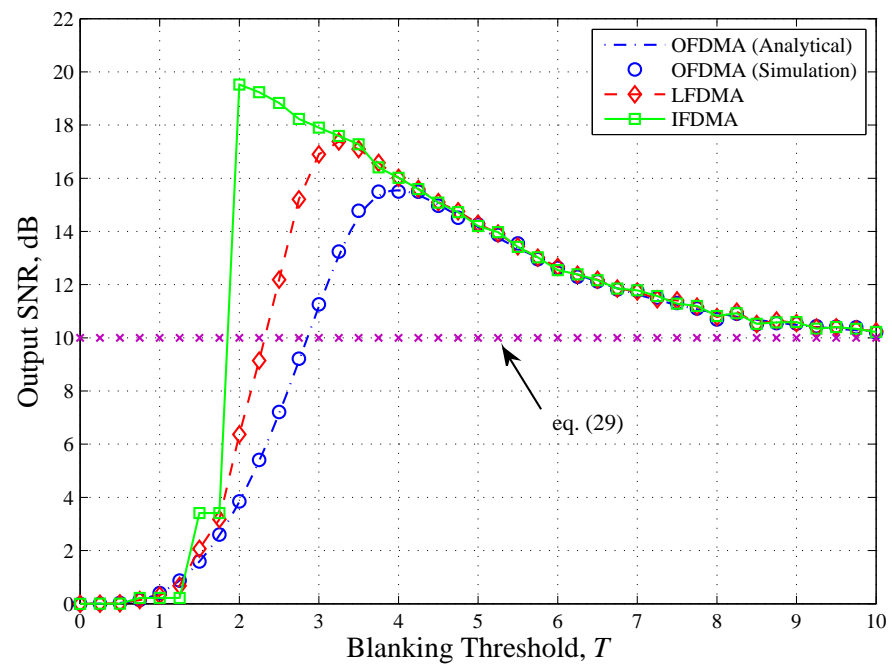

Fig. 7: Output SNR of OFDMA, LFDMA and IFDMA versus blanking threshold for SINR $=-10 \mathrm{~dB}, p=0.01$ and SNR $=40 \mathrm{~dB}$.

technique tries to minimize. From this figure it can be seen that the analytical results of OFDMA system, obtained from (20), are in good agreement with the simulation ones and this verifies the accuracy of our simulations. As anticipated, we can see that LFDMA performs better than OFDMA whereas IFDMA outperforms both systems. There is a general trend that when $T$ is too small $\{T \lesssim 2\}$ the system performance degrades dramatically for the three systems since a great amount of the useful signal energy is lost. On the other hand, if $T$ is too high $\{T \rightarrow \infty\}$ no blanking takes place which allows all the IN energy to be part of the detected signal. In such scenario, the output SNR approaches $10 \mathrm{~dB}$ as can clearly be observed from this figure and it is mathematically expressed as

$$
\operatorname{SNR}_{O / L / I F D M A}(T \rightarrow \infty)=10 \log _{10}\left(\frac{\sigma_{\bar{x}}^{2}}{\sigma_{w}^{2}+p \sigma_{i}^{2}}\right)
$$

when $p \sigma_{i}^{2} \gg \sigma_{w}^{2}$, (29) can be approximated to

$$
\simeq 10 \log _{10}\left(\frac{1}{p \sigma_{i}^{2}}\right)
$$

It is also interesting to observe that for each system there exists an OBT that maximizes the output SNR. It is important to highlight the fact that for the OFDMA and LFDMA systems the performance gradually improves before it reaches the optimal value whereas for the IFDMA the performance sharply improves at blanking threshold of about 2 . The optimization of the blanking threshold for the three systems is investigated next.

\section{BLANKING THRESHOLD OPTIMIZATION AND MAXIMUM ACHIEVABLE OUTPUT SNR}

In this section, the OBT that maximizes the output SNR for the three systems under different IN conditions is studied and determined by satisfying the following argument

$$
T_{o p t}=\arg \max _{0 \leq T<\infty}\left\{\mathrm{SNR}_{O / L / I F D M A}\right\}
$$




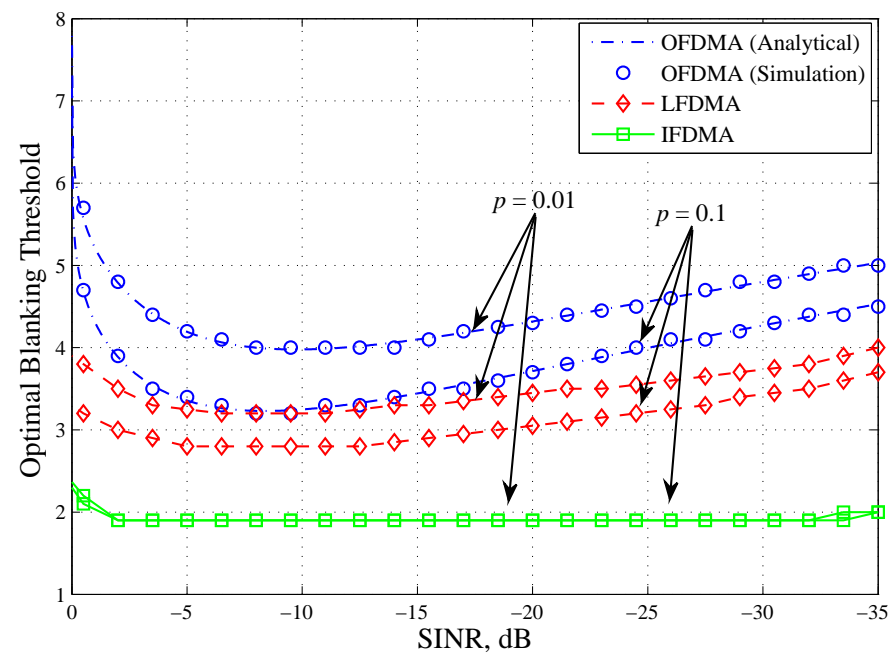

Fig. 8: Optimal blanking threshold of OFDMA, LFDMA and IFDMA versus SINR for $p=0.01$ and $0.1, \mathrm{SNR}=40 \mathrm{~dB}$.

Fig. 8 illustrates the OBT versus SINR for the OFDMA, LFDMA and IFDMA systems with two impulse probabilities $p=0.01$ and $p=0.1$. The analytical results of the OFDMA system are obtained from (19) and it is clear that these results closely match the simulations. For OFDMA and LFDMA, it is noticeable that as $p$ increases the OBT is decreased. Interestingly enough, in the case of IFDMA, it is clear that the OBT is independent of IN parameters. This implies that if the IFDMA scheme is employed with blanking, it becomes possible to optimally blank IN without the need for any IN estimations. Consequently, IN estimation errors can be avoided and also the receiver complexity will be significantly reduced.

The maximum achievable output SNR corresponding to the OBT found above is shown in Fig. 9 versus SINR when $p=0.01$ and 0.1 for the three systems. To provide a comparative analysis, we have also added the SNR performance of the conventional system (29), i.e. without the blanking device. The analytical results of the OFDMA system are obtained by substituting (19) into (21) and then into (20) whereas the for the conventional system the results are obtained from (29). It is obvious that the absence of the blanking device will result in a significant loss in the output SNR and this loss becomes more severe as the IN frequency of occurrence is increased. As expected, it is shown that LFDMA and IFDMA always outperform OFDMA for the given probabilities and that the IFDMA system attains the best performance. To highlight the improvement, we have plotted the relative gain $\left(G_{R}\right)$ versus SINR in Fig. 10. This gain is defined as the improvement in the output SNR obtained by LFDMA and IFDMA over the OFDMA and is given by

$$
G_{R}=10 \log _{10}\left(\frac{\operatorname{SNR}_{L / I F D M A}\left(T=T_{o p t}\right)}{\operatorname{SNR}_{O F D M A}\left(T=T_{\text {opt }}\right)}\right)
$$

It is obvious that the largest enhancement is achieved in the intermediate SINR region $(-5 \mathrm{~dB} \rightarrow-15 \mathrm{~dB})$. Under the same IN conditions, it is shown that IFDMA always provides more gain compared to the LFDMA system. For instance, there is a gain of up to about $4 \mathrm{~dB}$ in case of IFDMA over using OFDMA system at SINR $=-9$ and $p=0.01$, whereas about

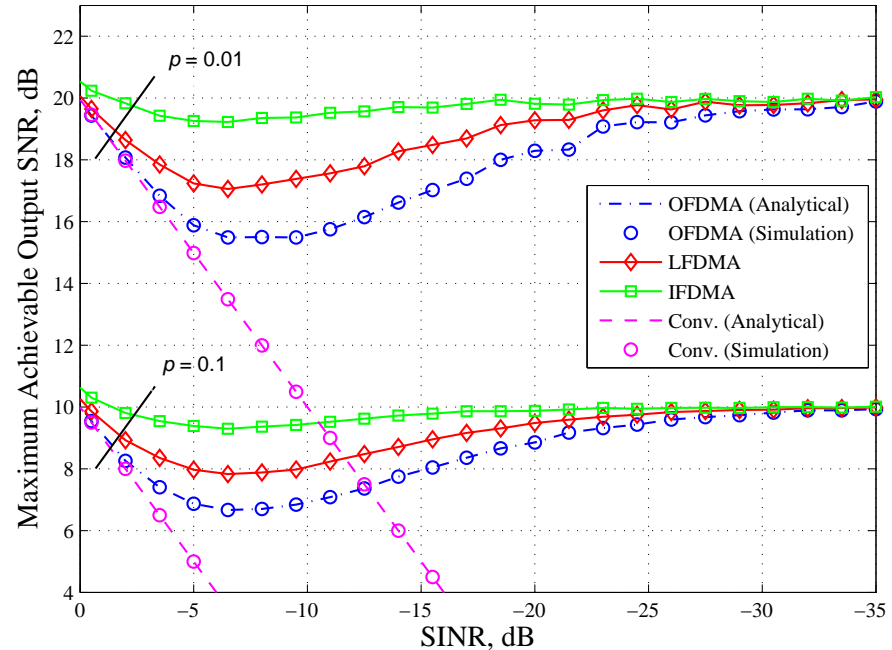

Fig. 9: Maximum achievable output SNR of OFDMA, LFDMA and IFDMA and conventional systems versus SINR for $p=0.01$ and 0.1 , $\mathrm{SNR}=40 \mathrm{~dB}$.

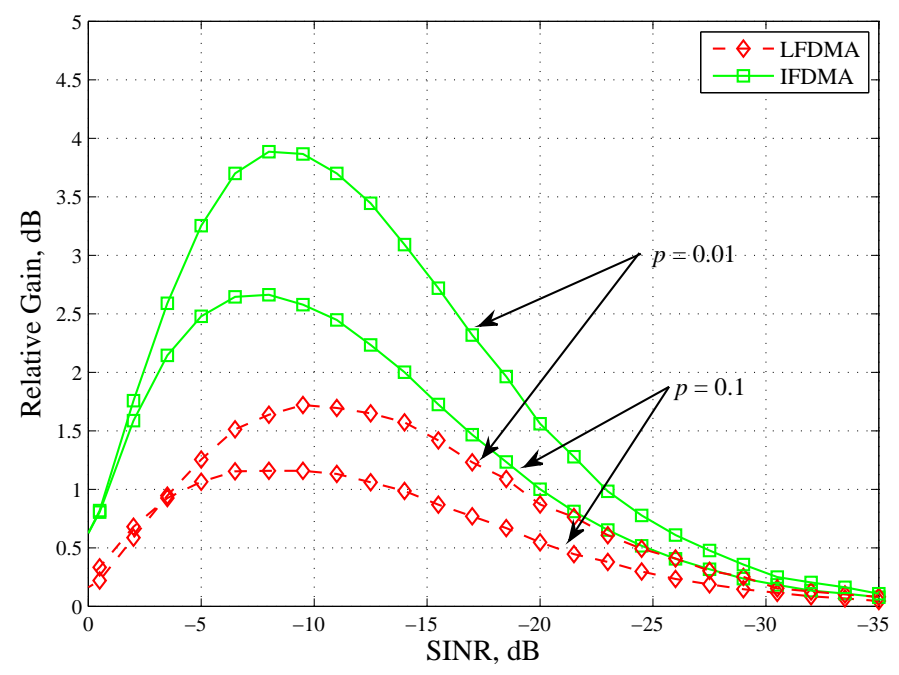

Fig. 10: The SNR gain of LFDMA and IFDMA systems relative to the OFDMA system versus SINR for $p=0.01$ and $0.1, \mathrm{SNR}=40 \mathrm{~dB}$.

$2.75 \mathrm{~dB}$ enhancement is attained with the LFDMA system at SINR $=-10 \mathrm{~dB}$ for the same impulse probability. Furthermore, it is worthwhile mentioning that even in a heavily disturbed IN environment $(p=0.1)$ gains of up to $2.5 \mathrm{~dB}$ and $1.75 \mathrm{~dB}$ can be obtained for LFDMA and IFDMA, respectively.

\section{Conclusion}

In this paper we have investigated the performance of SCFDMA with optimized blanking in the presence of IN. It was demonstrated that the proposed system is able to outperform the conventional OFDMA blanking-based technique in terms of minimized probability of blanking error and an increase in the output SNR which can be up to $4 \mathrm{~dB}$. In addition, and most importantly, it was found that the application of IFDMA makes it feasible to optimally blank IN without the need for prior knowledge about the noise characteristics. Although only blanking was considered in this paper, SC-FDMA can be applied conjointly with other nonlinear preprocessors such as clipping and hybrid (combined blanking and clipping). 


\section{REFERENCES}

[1] M. Hazen, "The technology behind HomePlug AV powerline communications," Computer, vol. 41, no. 6, pp. 90-92, 2008.

[2] X. Cheng, R. Cao, and L. Yang, "Relay-aided amplify-and-forward powerline communications," IEEE Trans. Smart Grid, vol. 4, no. 1, pp. 265-272, March 2013.

[3] M. Zimmermann and K. Dostert, "A multipath model for the powerline channel," IEEE Trans. Commun., vol. 50, no. 4, pp. 553-559, 2002.

[4] — "Analysis and modeling of impulsive noise in broad-band powerline communications," IEEE Trans. Electromagn. Compat., vol. 44, pp. 249258, Feb. 2002.

[5] O. Hooijen, "A channel model for the residential power circuit used as a digital communications medium," IEEE Trans. Electromagn. Compat., vol. 40, no. 4, pp. 331-336, 1998.

[6] M. G. Sanchez, L. de Haro, M. C. Ramon, A. Mansilla, C. M. Ortega, and D. Oliver, "Impulsive noise measurements and characterization in a UHF digital TV channel," IEEE Trans. Electromagn. Compat., vol. 41, no. 2, pp. $124-136$, May 1999.

[7] D. Anastasiadou and T. Antonakopoulos, "Multipath characterization of indoor power-line networks," IEEE Trans. Power Del., vol. 20, no. 1, pp. 90-99, Jan. 2005.

[8] H. Meng, Y. L. Guan, and S. Chen, "Modeling and analysis of noise effects on broadband power-line communications," IEEE Trans. Power Del., vol. 20, no. 2, pp. 630-637, Apr. 2005.

[9] P. Cuntic and A. Bazant, "Analysis of modulation methods for data communications over the low-voltage grid," in Proc. Int. Conf. Telecommun., vol. 2, Jun. 2003, pp. 643-648.

[10] Y. H. Ma, P. L. So, and E. Gunawan, "Performance analysis of OFDM system for broadband power line communications under impulsive noise and multipath effects," IEEE Trans. Power Del., vol. 20, no. 2, pp. 674 682, Apr. 2005.

[11] M. Gotz, M. Rapp, and K. Dostert, "Power line channel characteristics and their effect on communication system design," IEEE Commun. Mag., vol. 42, no. 4, pp. 78-86, 2004.

[12] D. Middleton, "Canonical and quasi-canonical probability models of class A interference," IEEE Trans. Electromagn. Compat., vol. EMC-25, pp. 76-106, May 1983

[13] _ - "Statistical-physical models of electromagnetic interference," IEEE Trans. Electromagn. Compat., vol. EMC-19, pp. 106-127, Aug. 1977.

[14] J. A. C. Bingham, "Multicarrier modulation for data transmission: An idea whose time has come," IEEE Commun. Mag., vol. 28, no. 5, pp. 5-14, May 1990.

[15] E. Del Re, R. Fantacci, S. Morosi, and R. Seravalle, "Comparison of CDMA and OFDM techniques for downstream power-line communications on low voltage grid," IEEE Trans. Power Del., vol. 18, no. 4, pp. 1104-1109, Oct. 2003.

[16] O. P. H. et al., "Detection and removal of clipping in multicarrier receivers," Eur. patent Appl. EP1043874, Oct. 2000, Bull. 20000/41.

[17] N. P. Cowley, A. Payne, and M. Dawkins, "COFDM tuner with impulse noise reduction," Eur. Patent Appl. EP1180851, Feb. 2002.

[18] S. V. Zhidkov, "On the analysis of OFDM receiver with blanking nonlinearity in impulsive noise channels," in Proc. Int. Symp. Intell. Signal process. Commun. Syst., Nov. 2004, pp. 492-496.

[19] K. S. Vastola, "Threshold detection in narrow-band non-gaussian noise," IEEE Trans. Commun., vol. COM-32, no. 2, pp. 134-139, Feb. 1984.

[20] S. V. Zhidkov, "Performance analysis and optimization of OFDM receiver with blanking nonlinearity in impulsive noise environment," IEEE Trans. Veh. Technol., vol. 55, no. 1, pp. 234-242, Jan. 2006.
[21] E. Alsusa and K. Rabie, "Dynamic peak-based threshold estimation method for mitigating impulsive noise in power-line communication systems," IEEE Trans. Power Del., vol. 28, no. 4, pp. 2201-2208, Oct. 2013.

[22] K. Rabie and E. Alsusa, "Preprocessing based impulsive noise reduction for powerline communications," to appear in IEEE Trans. Power Del., 2013.

[23] H. Myung, J. Lim, and D. Goodman, "Single carrier FDMA for uplink wireless transmission," IEEE Veh. Technol. mag., vol. 1, no. 3, pp. 30-38, 2006.

[24] N. Benvenuto, R. Dinis, D. Falconer, and S. Tomasin, "Single carrier modulation with nonlinear frequency domain equalization: An idea whose time has come - again," Proc. IEEE, vol. 98, no. 1, pp. 69-96, 2010.

[25] M. Ghosh, "Analysis of the effect of impulse noise on multicarrier and single carrier QAM systems," IEEE Trans. Commun., vol. 44, no. 2, pp. 145-147, Feb. 1996.

[26] R. van Nee and R. Prasad, "OFDM for wireless multimedia communications," Boston, MA, USA: Artech House, 2000.

[27] H. Myung, J. Lim, and D. Goodman, "Peak-to-average power ratio of single carrier FDMA signals with pulse shaping," in Proc. IEEE Personal, Indoor and Mobile Radio Commun., Sep. 2006, pp. 1-5.

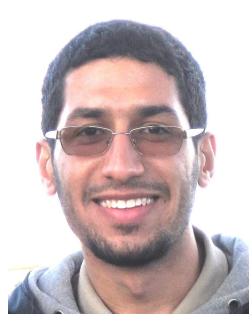

Khaled Maaiuf Rabie (S'12), received the B.Sc. degree (Hons.) in electrical and electronic engineering from the University of Tripoli, Tripoli, Libya, in 2008 and the M.Sc. degree (Hons.) in communication engineering from the University of Manchester, Manchester, UK, in 2010. Since September 2011, he has been working toward the Ph.D. degree in the Microwave and Communications Systems (MACS) group of the University of Manchester. His current research interests are power-line communications including power-line channel modeling, interference and impulsive noise mitigation for smart-grid applications as well as multipleinput multiple-output (MIMO) systems.

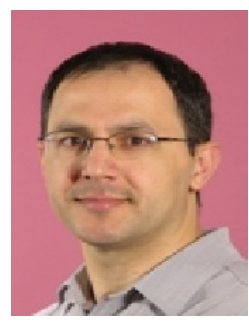

Emad Alsusa (M'06-SM'07) received a Ph.D. degree in electrical and electronic engineering from Bath University, Bath in 2000. Following his $\mathrm{PhD}$ he joined the School of Engineering and Electronics at Edinburgh University as a MobileVCE Postdoctoral Research Fellow, working on industrially led projects on link enhancement techniques for future high data rate wireless communication systems. In 2003, he joined the University of Manchester as an academic member of the school of Electrical and Electronic Engineering, where he lectures on communication engineering subjects. His research interests include signal processing and analysis of wireless communication networks, with particular focus on modulation and multiple access, channel estimation, coding, interference mitigation, multiuser detection, MIMO techniques and spectrum sensing techniques for cognitive radio applications. He served as a technical program committee member on numerous IEEE flagship conferences and chaired the Manchester EEE postgraduate conference in 2010 . 